\title{
Experimental study on the effect of longitudinal magnetic field on Townsend discharge characteristics in low pressure argon gas
}

\author{
Sabri J. Mohammed ${ }^{1}$, Mohammed K. Khalaf ${ }^{2}$, Muataz A. Majeed ${ }^{1,3, *}$, Hanaa E. Jasem ${ }^{1}$ \\ ${ }^{1}$ Department of Physics, Faculty of Education for Pure Sciences, Tikrit University, Tikrit, Iraq \\ 2Ministry of Science and Technology, Baghdad, Iraq \\ 3Ministry of Education, Baghdad, Iraq
}

\section{A R T I C LE IN F O}

\section{Article history:}

Received 16 September 2016

Received in revised form

31 January 2017

Accepted 1 February 2017

\section{Keywords:}

External longitudinal magnetic field

Paschen curves

Minimum breakdown voltage

Townsend coefficient

Ionization efficiency

\begin{abstract}
A B S T R A C T
In this paper the electrical breakdown has been described for low-pressure argon discharges under the influence of an external longitudinal magnetic field. Plane-parallel stainless steel electrodes $(7.25 \mathrm{~cm}$ diameter $)$ are sustained with a dc voltage $(0<\mathrm{V}<1 \mathrm{kV})$, the pressure was varied between 0.4 mbar and $2.6 \mathrm{mbar}$ and the inter-electrode distance $2.0 \mathrm{~cm}$. A Helmholtz coil was used to produce a uniform magnetic field $(B=270 G)$ from an inductive copper coil of diameter $(1 \mathrm{~mm})$. A Paschen curve has been obtained and the first Townsend ionization coefficient $(\alpha)$, the secondary electron emission coefficient $(\gamma)$ and the ionization efficiency $(\eta)$ were plotted with respect to the variation of the reduced field $(E / P)$. The calculated value of the $\eta(E / p)$ and $\gamma(\mathrm{E} / \mathrm{p})$ inside the magnetic trap is in good agreement with the previous literatures results.
\end{abstract}

(C) 2017 The Authors. Published by IASE. This is an open access article under the CC BY-NC-ND license (http://creativecommons.org/licenses/by-nc-nd/4.0/).

\section{Introduction}

The interest in studying the magnetic field effect on the characteristics of electrical breakdown and on the properties of a Townsend discharge is motivated by a necessity of gaining a better understanding of the complex mechanisms of gas discharge phenomena and also because the B-field may contribute favorably for dealing with practical problems associated with the use of this kind of discharge for plasma processing technologies. These researches included the study of magnetizes plasma discharge at different working parameters such as the pumping gases, the materials and the geometry of electrodes, gas pressure, chamber temperature, humidity of discharge chamber and the type of applied voltage (Wais et al., 2001).

The electrical breakdown is presented by Petraconi et al. (2004) at low-pressure of argon and nitrogen gases under the influence of an external longitudinal magnetic field. Plane-parallel aluminum electrodes is used at various spacing $(4 \mathrm{~cm}$ $<\mathrm{d}<11 \mathrm{~cm})$ with a dc voltage $(0<\mathrm{V}<1 \mathrm{kV})$. A measurement of the secondary electron-emission

\footnotetext{
* Corresponding Author.

Email Address: Muataz.amj2020@gmail.com (M. A. Majeed) https://doi.org/10.21833/ijaas.2017.02.016

2313-626X/C 2017 The Authors. Published by IASE.

This is an open access article under the CC BY-NC-ND license

(http://creativecommons.org/licenses/by-nc-nd/4.0/)
}

coefficient $\gamma$ from $\mathrm{MgO}$ film in a plasma display panel is carried out by Uhm et al. (2001). The influence of the secondary electron emission on the breakdown voltage in micro discharges on the plasma display panel is investigated by making use of the Townsend sparking criterion. Auday et al. (1998) presented a experimental study of the effective secondary emission coefficient for rare gases and copper electrodes. The measurements are made for variations of the effective secondary emission coefficient with reduced field $E / p$ at different parallel plane electrodes spacing $(2 \mathrm{~mm}<$ $\mathrm{d}<1 \mathrm{~cm}$ ). In fact, as the experimental results show, this phenomenon was confirmed by the reduction of the breakdown voltage when a magnetic field was applied. This study contributes Townsend discharge regime at a new range of plane-parallel electrode separations $(2 \mathrm{~cm})$ and different working pressures. Depending upon the energy range level, which can be delivered by these pulsed discharges, their pulse durations and modes of operation, a number of research interests has been developed for applications in medicine (Woloszko et al., 2002; Abou-Ghazala et al., 2002; Nunnally et al., 2005; Akiyama et al., 2007). Helmholtz coils are two identical circular coils of wire with identical electrical currents flowing in the same direction and arranged symmetrically. The coils have a common axis such that the magnetic field of each adds to the magnetic field of the other. They are placed a distance apart equal to the radii of the coils. In this 
arrangement, the magnetic field can be shown from theory and measurements to be nearly uniform in the center and so can be used to locate experimental apparatus requiring a uniform magnetic field. This arrangement of coils is named in honor of the German physicist Hermann von Helmholtz.

In this experiment the magnetic field strength for a pair Helmholtz coils will is derived as a function of the axial distance between and outside the coils. The result will then be compared to actual measurements of the magnetic field. The magnetic field measured transversely to the center of the coils will also be determined. The law is named for Jean-Baptiste Biot and Felix Savart, who first formulated this relationship in 1820. Using the principle of symmetry, the Biot-Savartlaw will be used in this experiment to determine the magnetic field strength as a function of distance along the axis of a single circular coil of wire carrying current $i$. The result will be used to determine the magnetic field of a pair of Helmholtz coils along their axis of symmetry. The Biot-Savart law can be better understood and applied by considering Fig. 1, which depicts a randomly-shaped wire with a steady current i flowing in it. According to the Biot-Savart law, the magnetic field resulting from the current flowing in a small length of the wire at some arbitrary distance $r$ away is given in the Eq. 1 by Parks (2013):

$\Delta B=\frac{\mu_{o} i}{4 \pi} \frac{\Delta l \sin \theta}{r^{2}}$

where, $\mu_{0}$ is permeability of free space,$N$ number of turns of wire on each coil, I the current flowing in each coil, $\mathrm{R}$ the radius of each of the coils.

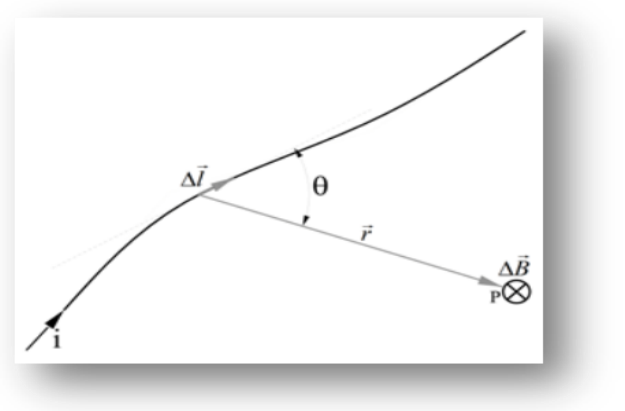

Fig. 1: The incremental magnetic field strength, at point $P$ is given by the Biot-Savart law

The glow discharge system was investigated by Raizer (1991). The easiest species of glow discharge is a direct-current glow discharge. In its form, it includes of two electrodes in a cell held at low pressure ( 0.1 - 10 Torr). The cell is typically overfilled with neon, but other gases can also be used (Tan et al., 2006; Ledernez et al., 2009; Georghiou et al., 2005). In this work, we have developed an experimental device to observe the effect of a magnetic field parallel to the electric field on the gas breakdown voltage. We expected the lateral diffusion of electrons to be hindered by the magnetic field consequently reducing losses and enhancing the ionization efficiency in the Townsend regime.

\section{Materials and methods}

Fig. 2 shows a photograph of the main experimental set-up used in this work. On the righthand side one sees the plasma chamber: Pyrex glass cylindrical vacuum chamber has been used for plasma discharge with length and diameter of 35 $\mathrm{cm}$ and $14.5 \mathrm{~cm}$ respectively. Basically, the inside of the chamber consists of two electrodes between which the breakdown - discharge is formed, the electrodes are made of stainless steel material and each of them was $7.25 \mathrm{~cm}$ diameter and $3 \mathrm{~mm}$ thickness. The Plane-parallel electrodes are shielding by insulator disk. The application of an induction magnetic field (produced by the Helmholtz coil) with intensity of the order of 270 Gauss is effective in confining of the elections and is negligible in the case of ions. This demands that the diameter of the induction coil must coincide with the outer diameter of the discharge tube. This device was made up of two parallel coaxial circular coils (200 turns in each loop, wound in series) which were separated from each other by a distance equal to the radius of one coil $(14.5 \mathrm{~cm})$. The windings of our Helmholtz coil was made of standard $1 \mathrm{~mm}$ wire to provide the maximum possible current. The coil was powered by a variable dc power supply $(50 \mathrm{~V}$, 12Amp.). The magnetic flux density near the discharge electrode could be changed by increasing the pass current through the induction coil. The measurement of the magnetic field distribution is performed using a Hall's probe and NV621 Tesla meter. The influence of longitudinal magnetic fields on the breakdown voltage of argon gas plasma at low pressures $(0.4$ 2.6) mbar have been investigated The experimental measurements at constant interelectrode spacing $2 \mathrm{~cm}$, are recorded at room temperature $\left(\mathrm{T}=2{ }^{\circ} \mathrm{C}\right)$ and humidity of about $(50-55$ $\%$ ). The voltage-current characteristics can be established with the scheme shown in Fig. 3. The current-limiting resistor $(300 \mathrm{k} \Omega)$ permits limiting the I-V characteristics in Townsend discharge region and varying the resistance in the external electrical circuit so that the current can be independently controlled at any voltage level.

\section{Results and discussion}

Breakdown voltage and Paschen curves: The gas breakdown voltage $V_{B}$ was determined as a function of the product $P d$. The measurements were made for argon discharge the pressure was varied between $0.4 \mathrm{mbar}$ and $2.6 \mathrm{mbar}$ and the inter-electrode distance 2.0, the application of an induction magnetic field with intensity of the order of 270 Gauss. The Paschen curve relates the breakdown voltage over a gap as function of the pressure $\mathrm{P}$ and the length of the gap d. Fig. 4 shows the mean 
Paschen curves for argon discharges with and without applying the longitudinal magnetic field. Georghiou et al. (2005) showed the Paschen curves are plane-parallel gap-dependent. Accordingly, the breakdown voltage is reduced as shown in Fig. 4.

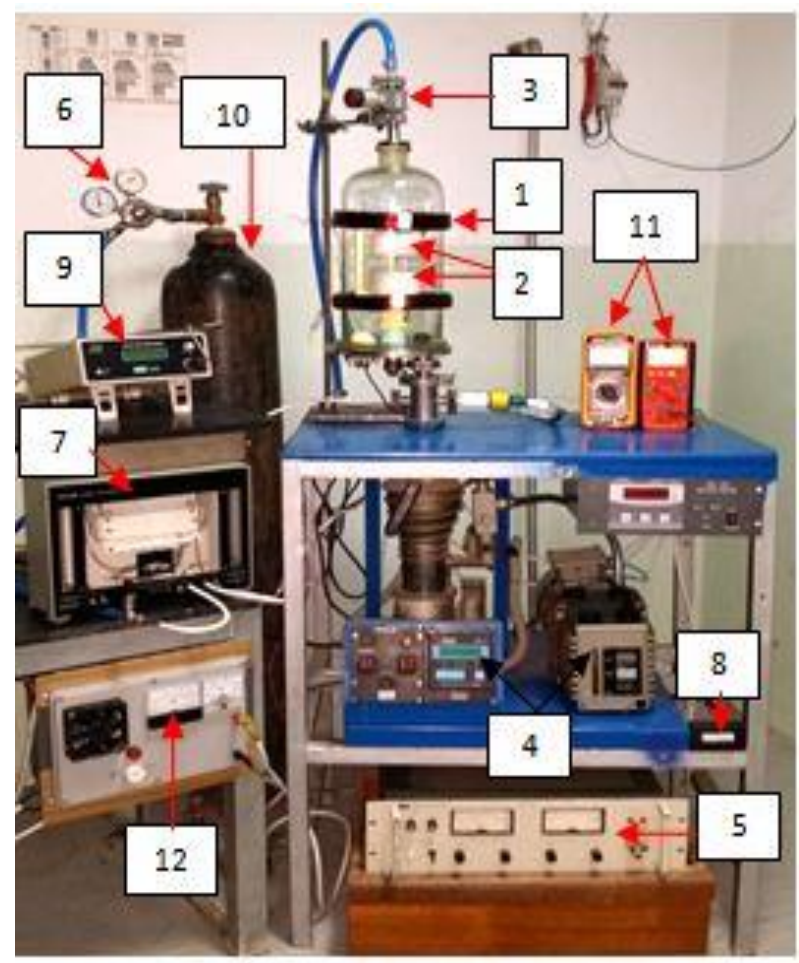

Fig. 2: A photograph of the main experimental set-up used in this work.(1) Helmholtz coil (2) plasma chamber((a) cathode (b) anode )) (3) needle valve (1-160sccm) current limited discharge resistance (4) vacuum system (5) power supply (Helmholtz coil) (6) control gas (7) flow meter and gas mixer (8) current limited resistance (9) Hall probe (10) bottles gas (11) ammeter and voltmeter (12) H.V power supply.

In this work, a significant downward of Paschen curves is observed under the influence of longitudinal magnetic field especially in the region of Paschen's minimum because of the higher efficiency of the secondary ionization processes in argon plasma discharge. The experimental results showed a fast decreasing of the breakdown voltages on the left side of the minimum Paschen curves and gradually increasing on the right side as $\mathrm{Pd}$ is increased. This can be attributed to the increase of collision frequency on the left side and decreasing the ionization cross section on the right side for which more energy is required for electrons to reach the breakdown voltage (Wais et al., 2001).

Variation of $\eta$ with E/P: The influence of external longitudinal magnetic field on the variation of ionization efficiency coefficient $\eta$ with reduced electric field is shown in Fig. 5. The effect of longitudinal magnetic field becomes more significant on the behavior of $\eta$ versus E/P. At low working pressure; the number of collision between the released electrons with the argon molecules is decreased thus the confinement of electrons by the magnetic field is enhanced the values of $\eta$ as the reduced electric field is decreased.

Fig. 5 shows the variation of the ionization efficiency with E/P for argon gas we observe from these figures that $\eta(\mathrm{E} / \mathrm{P})$, at small $\mathrm{E} / \mathrm{P}$; increases sharply, but then reaches a maximum and falls off on further increase of $\mathrm{E} / \mathrm{P}$.

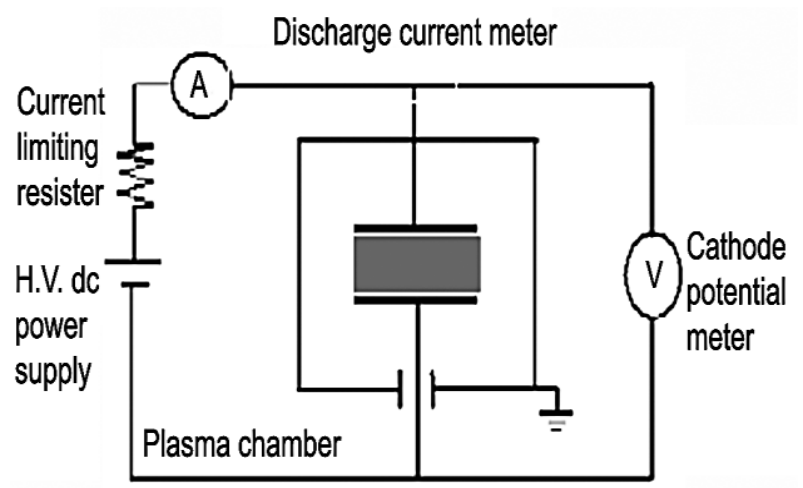

Fig. 3: The dc discharge circuit of I-V characteristics

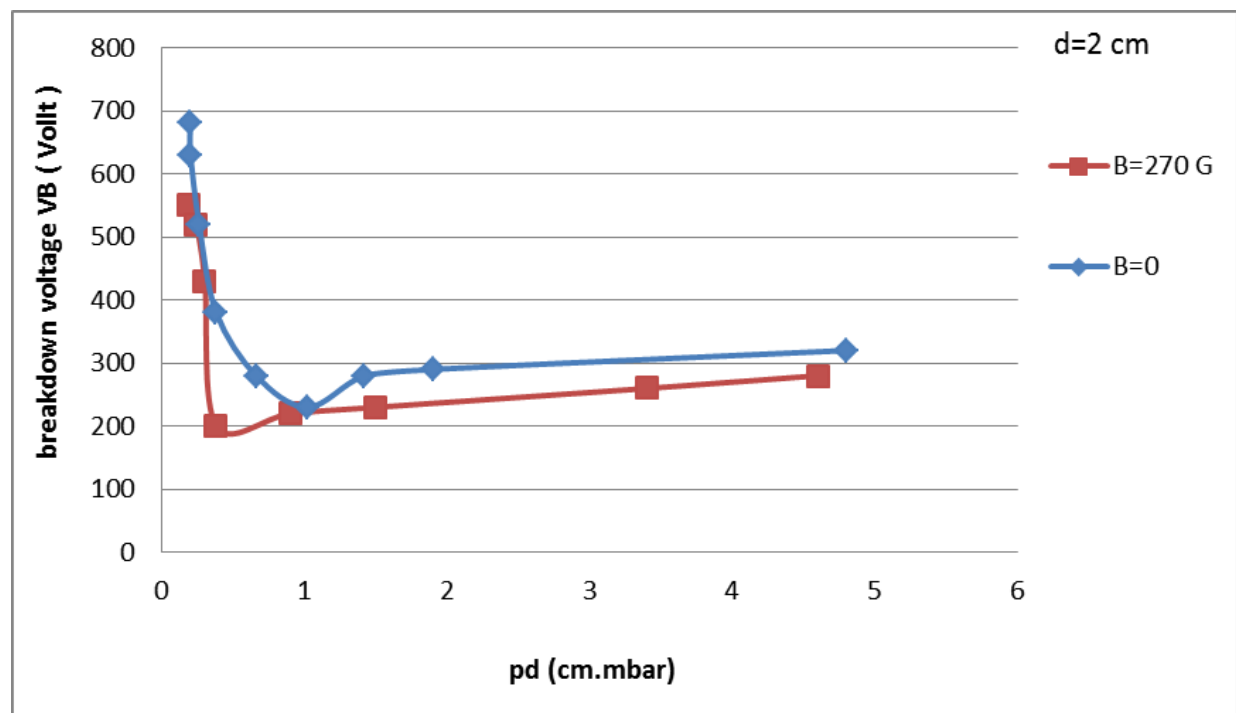

Fig. 4: Paschen curves for argon discharges at inter-electrode spacing $d=2 \mathrm{~cm}$ with and without applying the external longitudinal magnetic field intensity 


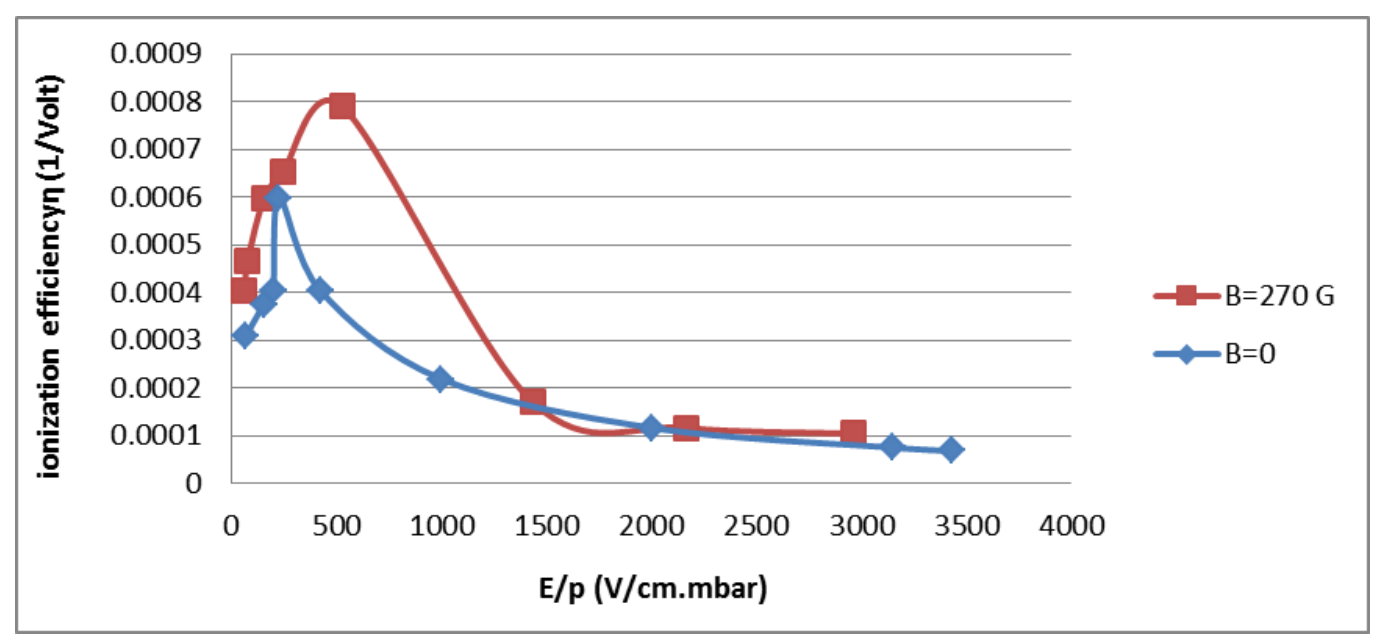

Fig. 5: The variation of ionization efficiency coefficient $\eta$ versus $E / P a t$ inter-electrode spacing $d=2 \mathrm{~cm}$ with and without applying the external longitudinal magnetic field.

The maximum value of $\eta$ that reached in this study was $0.025 \mathrm{~V}^{-1}$ while the actual value is $\eta_{\max }=0.00075 \mathrm{~V}^{-1}$ therefore only the descending branch of $\eta$ is obtained. In the absence of magnetic field, the required voltage for maintaining the breakdown discharge depends on the work function of cathode material for which a high voltage should be supplied if the cathode is made of high material work function. The present of magnetic field lengthens the trajectory of free electrons (i.e. Larmor frequency) in ionization region and decreased the mean energy of released electrons to reach anode which in turn increases the number of collisions (Burm, 2008).

Variation of $\gamma$ with E/P: The variation of the effective secondary electron emission coefficient $\gamma$ with the reduced field E/P is shown in Fig. 6. The values of secondary electron emission coefficients $\gamma$ are calculated from the mean Paschen curves. In this work, at small inter-electrode spacing and high reduced electric field, the confinement of released electrons is enhanced by the longitudinal magnetic field. Therefore, the associated effect of magnetic field increases the values of $\gamma$ as the reduced electric field is increased. For high values of $\mathrm{E} / \mathrm{p}, \gamma$ rises more strongly in the present work than in the literature. This could arise from the excitation within the gas becomes greater than ionization as indicated by the low values of the ionization efficiency coefficient $\eta$. For small value of $E / p$, the electrons are released from the cathode by a resonant photons emission (photoelectric effect). As d increases, this process loses importance since any photon emitted has less chance of striking the cathode. Therefore, for low fields the coefficient $\gamma$ also depends on the value chosen for d (Naidu and Kamaraju, 2009).

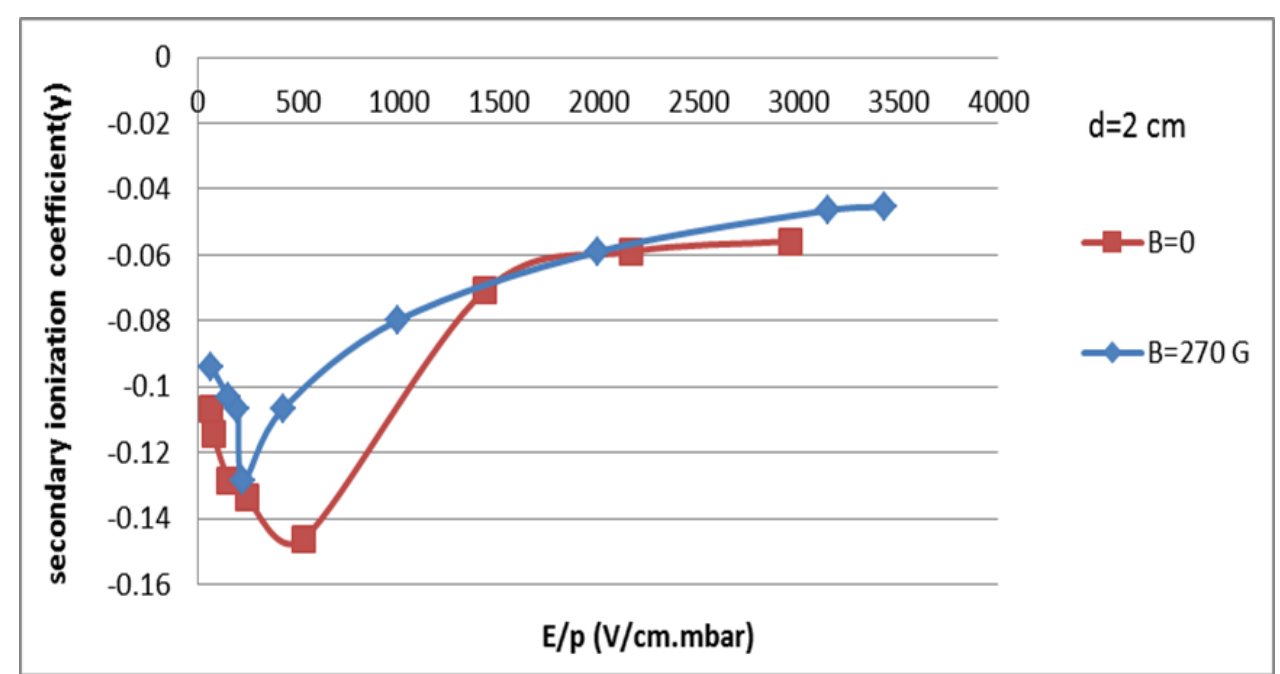

Fig. 6: The variation of secondary electron emission coefficient $\gamma$ versus $\mathrm{E} / \mathrm{P}$ at inter-electrode spacing $\mathrm{d}=2 \mathrm{~cm}$ with and without applying the external longitudinal magnetic field

\section{Conclusion}

We have investigated the influence of a longitudinal magnetic field on the Paschen curves and on the Townsend parameters. We observed that the magnetic field applied along the discharge axis promoted a reduction of the breakdown voltage. The breakdown is facilitated by the magnetic confinement of electrons which reduces the electron losses and effectively increases the collision frequency between electrons and the gas particles at a given reduced field, thus increasing the ionization 
efficiency. This effect is equivalent to a change of the operating gas by another of lower ionization potential. The presence of the magnetic field enhances the secondary ionization coefficient at a given $E / P$ value.

\section{Acknowledgment}

This work has been financial supported by University of Tikrit and College of Education of Pure Sciences. We thank the Ministry of Sciences and Technology supporting this work and providing laboratory services.

\section{References}

Abou-Ghazala A, Katsuki S, Schoenbach KH, Dobbs FC, and Moreira KR (2002). Bacterial decontamination of water by means of pulsed-corona discharges. IEEE Transactions on Plasma Science, 30(4): 1449-1453.

Akiyama H, Sakugawa T, Namihira T, Takaki K, Minamitani Y, and Shimomura N (2007). Industrial applications of pulsed power technology. IEEE Transactions on Dielectrics and Electrical Insulation, 14(5): 1051-1064.

Auday G, Guillot P, Galy J, and Brunet H (1998). Experimental study of the effective secondary emission coefficient for rare gases and copper electrodes. Journal of Applied Physics, 83(11): 5917-5921.

Burm K (2008). Breakdown magnetic field in an inductively coupled plasma. Physics Letters A, 372(41): 6280-6283.

Georghiou GE, Papadakis AP, Morrow R, and Metaxas AC (2005). Numerical modelling of atmospheric pressure gas discharges leading to plasma production. Journal of Physics D: Applied Physics, 38(20): R303-R328.
Ledernez L, Olcaytug F, Yasuda H, and Urban G (2009). A modification of Paschen law for Argon. 29th ICPIG, Cancun, Mexico, 19: 2059-2077.

Naidu K and Kamaraju V (2009). High voltage engineering. McGraw-Hill, $4^{\text {th }}$ Edition, New Delhi, India.

Nunnally WC, Lewis R, Allen F, Hawkins S, Holmes C, Sampayan S, and Caporaso G (2005). Experiments with UV laser triggered spark gaps in a stacked Blumlein system. In the IEEE Pulsed Power Conference, IEEE: 1376-1381. https://doi.org/10.1109 /PPC.2005.300643

Parks JE (2013). Helmholtz coils-uniform magnetic fields Department of Physics and Astronomy, The University of Tennessee, Knoxville, USA.

Petraconi G, Maciel HS, Pessoa RS, Murakami G, Massi M, Otani C, and Sismanoglu BN (2004). Longitudinal magnetic field effect on the electrical breakdown in low pressure gases. Brazilian Journal of Physics, 34(4B): 1662-1666.

Raizer YP (1991). Gas Discharge Physics. Spring-Verlag, New York, USA.

Tan IH, Ueda M, Dallaqua RS, De Moraes Oliveira R, and Rossi JO (2006). Magnetic field effects on secondary electron emission during ion implantation in a nitrogen plasma. Journal of Applied Physics, 100(3): 033303. https://doi.org/10.1063/1. 2201695

Uhm HS, Choi EH, and Cho GS (2001). Influence of secondary electron emission on breakdown voltage in a plasma display panel. Applied Physics Letters, 78(5): 592-594.

Wais SI, Mohammed RY, and Yousif SO (2001). Influence of Axial Magnetic Field on the Electrical Breakdown and Secondary Electron Emission in Plane-Parallel Plasma Discharge. World Academy of Science, Engineering and Technology, 5(8): 12261231.

Woloszko J, Stalder KR, and Brown IG (2002). Plasma characteristics of repetitively-pulsed electrical discharges in saline solutions used for surgical procedures. IEEE Transactions on Plasma Science, 30(3): 1376-1383. 